UVX 2010 (2011) 99-105

DOI: $10.1051 /$ uvx/2011014

(C) Owned by the authors, published by EDP Sciences, 2011

\title{
L'endommagement des optiques du LMJ : problématique, mécanismes et métrologie
}

\author{
L. Lamaignère ${ }^{1}$, R. Courchinoux ${ }^{1}$, J.C. Poncetta ${ }^{1}$ et $\mathrm{H}$. Bercegol ${ }^{2}$ \\ ${ }^{1}$ CEA-DAM Cesta, BP. 2, 33114 Le Barp, France \\ ${ }^{2}$ CEA-DSM Saclay, 91191 Gif-sur-Yvette, France
}

\begin{abstract}
Résumé. Cet article traite de la problématique de l'endommagement laser sur les installations de fortes puissances. La métrologie mise en ouvre pour mesurer la durée de vie des optiques en laboratoire mais également sur chaîne est exposée. Quelques mécanismes à l'origine de l'endommagement laser sont également présentés ainsi que des solutions mises en œuvre afin de réduire les niveaux d’endommagement.
\end{abstract}

\section{INTRODUCTION}

Le Laser MégaJoule (LMJ) est une grande installation en développement au CEA-CESTA (Centre d'Études Scientifiques et Techniques d'Aquitaine) situé près de Bordeaux. Son prototype, la Ligne d'intégration laser (LIL), fonctionne depuis 2002. L'équipement de ces deux lasers nécessite environ 10 000 grands composants optiques d'excellente qualité. Due à l'accumulation des défauts, en particuliers lors des quatre passages dans la section amplificatrice, l'architecture retenue requiert des composants haut de gamme.

La LIL est constituée de quatre faisceaux laser identiques, 176 pour le LMJ, produisant chacun $7500 \mathrm{~J}$ de lumière ultraviolette focalisée sur des cibles de quelques centaines de microns. Ces niveaux d'énergie lumineuse très élevés imposent d'utiliser des faisceaux dont la section est grande pour une installation optique de ce type. Les composants optiques placés sur chacun des faisceaux sont ainsi caractérisés par une section utile de $400 \mathrm{~mm} \times 400 \mathrm{~mm}$. La très haute tenue au flux laser et les performances optiques doivent être excellentes, pour ne pas dégrader la qualité des faisceaux laser.

L'endommagement est une des limites avec laquelle il faut composer pour optimiser le dimensionnement, le coût et la maintenance de ces installations [1]. Les caractéristiques de tenue au flux sont celles qui ont une importance du point de vue opérationnel. Tout endommagement sans conséquence sur la performance du tir ni sur la dégradation de l'optique lors des tirs suivants est considéré comme non pertinent. Ne sont retenus que les dommages qui sont susceptibles de croître lors des tirs successifs. Il n'existe pas aujourd'hui de moyens permettant de connaître la performance de tenue au flux sans créer d'endommagement. Les tests sont réalisés sur des échantillons ou des parties d'un composant. Ils ne sont pas strictement répétables, le composant testé étant détruit.

On distingue deux actions métrologiques particulières :

- l'étude de l'occurrence des dommages qui se traduit par la mesure de densité de dommages,

- l'étude de la croissance des dommages, pour les dommages surfaciques, via la détermination des fluences seuils et des coefficients de croissance.

Avec ces deux données d'entrée, ou lois d'endommagement, il est possible de déterminer la durée de vie d'une optique connaissant le profil d'emploi de l'installation. Toutefois, les mesures sont réalisées sur

This is an Open Access article distributed under the terms of the Creative Commons Attribution-Noncommercial License 3.0, which permits unrestricted use, distribution, and reproduction in any noncommercial medium, provided the original work is properly cited. 
des installations et avec des faisceaux différents de ceux de la LIL et du LMJ. Se pose alors la question de la représentativité des mesures. Les résultats établis se doivent d'être valables quels que soient les paramètres expérimentaux : des lois d'échelle sont extraites qui permettent le passage des conditions de laboratoire aux conditions opérationnelles. Elles prennent en compte la taille du composant, la surface du faisceau et la durée d'impulsion.

Ces mesures réalisées en laboratoire sont confrontées aux observations effectuées sur l'installation LIL. Elles servent également de données d'entrée pour les modèles d'endommagement «macroscopiques » développés et qui permettent de rendre compte de l'endommagement de la chaîne, plus précisément pour la partie ultraviolette. En effet, une observation des composants en silice de la fin de chaîne est réalisée via l'acquisition de photographies plein champ des optiques afin de suivre l'évolution des dommages et d'estimer l'énergie transmise jusqu'à la cible.

Des actions sont entreprises afin de comprendre et ainsi réduire l'endommagement de ces optiques. Les mécanismes d'endommagement sont étudiés via des défauts modèles, de type fractures, qui rendent compte des défauts rencontrés dans les optiques et à partir desquels des modèles d'endommagement laser à l'échelle microscopique, initiés sur des défauts, sont développés. Il s'agit dans ces cas-là d'un endommagement intrinsèque auquel peuvent se coupler des effets non-linaires, de type Kerr, pouvant provoquer des endommagements par autofocalisation des faisceaux dans les optiques. La connaissance de l'ensemble de ces mécanismes et leur modélisation permettent de rétroagir sur l'architecture des composants et de proposer des solutions réduisant les densités de défauts à l'origine de l'endommagement laser.

Dans cet article, la problématique de l'endommagement laser sur les installations de fortes puissances est décrite. La métrologie mise en œuvre pour mesurer la durée de vie des optiques en laboratoire mais également sur chaine est exposée. Enfin différents mécanismes à l'origine de l'endommagement laser sont également présentés avec quelques solutions déjà mises en œuvre afin de réduire les niveaux d'endommagement.

\section{MESURE DE L'AMORÇAGE DES DOMMAGES}

\subsection{Problématique}

La très bonne qualité d'une optique de grandes dimensions nécessite de traquer les rares défauts qui peuvent conduire à un endommagement. A titre d'exemple, la densité de défauts sur des lames de silice est de l'ordre de 0,01 défauts $/ \mathrm{cm}^{2}$ pour des fluences inférieures à $14 \mathrm{~J} / \mathrm{cm}^{2}$. Ramener à la surface d'un composant, cela consiste à chercher la dizaine de dommages de taille micrométrique sur plusieurs centaines de centimètres carrés. Pour cela une procédure spécifique a été développée, celle-ci est présentée au paragraphe suivant. Les défauts qui conduisent à un endommagement laser sont de différents types. Cela peut être des particules absorbantes issues des produits de polissage. Une bonne corrélation a été montrée entre la concentration en cérium et le niveau d'endommagement des optiques [2]. Ces particules pouvant se retrouver dans les produits de polissage, il est alors nécessaire de réduire leur concentration. L'absorption du faisceau laser par de telles particules absorbantes a été modélisée et validée expérimentalement par Bonneau et collaborateurs [3] sur des matériaux modèles dans lesquels des nanoparticules d'or ont été implantées. Lorsque la concentration en particules absorbantes est largement réduite [4], la corrélation entre endommagement laser et concentration n'est plus valable. L'endommagement s'amorce alors à partir de fissures sous-surfaciques, elles-mêmes étant une conséquence des différentes étapes de polissage. Les travaux de Bercegol [5] permettent de rendre compte de l'amorçage des dommages à partir de fissures de taille nanométrique au niveau desquelles l'absorption du faisceau s'effectue. L'amélioration de la tenue au flux des surfaces des optiques passent alors par une réduction de la densité de fissures sous-surfaciques à travers l'amélioration des procédures de polissage ou par l'application de procédés qui réduiront leur nuisance. 


\subsection{Mesure de la densité de dommages}

Une méthode de test spécifique a été développée pour mesurer de très faibles densités de dommages sur de grandes dimensions. Cette méthode, nommée rasterscan, consiste en un balayage complet de l'optique afin d'illuminer tous les défauts qui peuvent conduire à un dommage. A l'issue du test, la densité de dommages est connue, pour l'optique testée, en fonction du niveau d'éclairement, donnée notée $D(F)$. Un exemple de résultat est reporté sur la figure 1. La procédure est décrite dans la référence [6] où une attention particulière est portée sur la détermination d'intervalles de confiance. En effet, les tests étant destructifs, il n'est pas possible de répéter la mesure sur le même composant, ce qui rend difficile la notion de répétabilité mais également la comparaison des mesures entre différentes installations. Ces intervalles de confiance sont donnés à deux écart-type. Ainsi, les résultats de tenue au flux de toute une série de composants, supposés identiques mais physiquement distincts, doivent être comprises dans $95 \%$ des mesures à l'intérieur de ces intervalles de confiance. Ceux-ci sont basés sur le nombre de dommages révélés lors du test. Cette démarche permet de s'assurer de la répétabilité de la mesure et a été mise en œuvre pour comparer des résultats obtenus sur différentes installations [7]. Cette procédure est utilisée pour la mesure de densités surfaciques de dommages et aussi pour la détermination des densités volumiques dans le cas d'un endommagement préférentiel dans la matière comme généralement observé pour les cristaux [8].

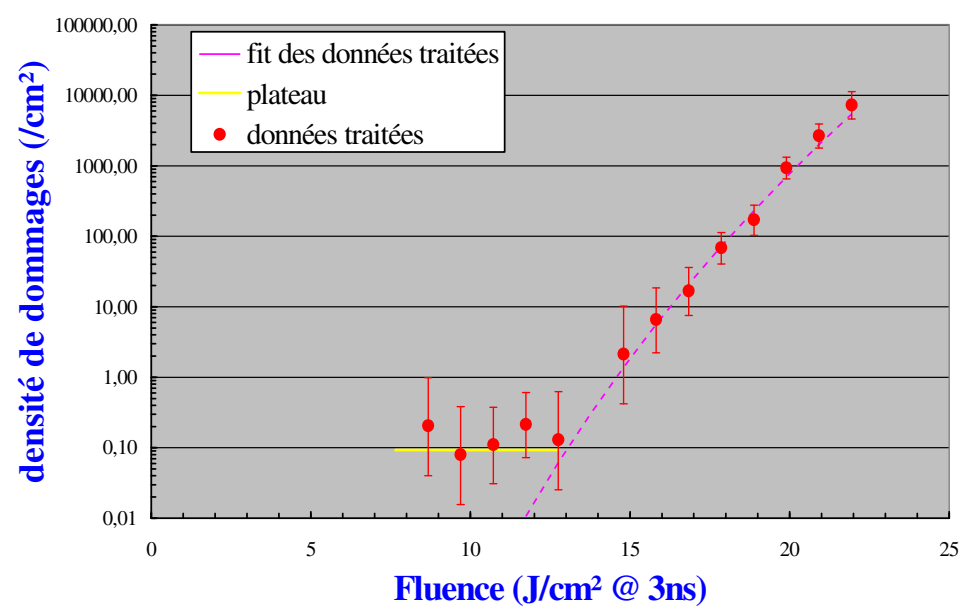

Figure 1. Densité de dommages en fonction de la fluence à l'issue d'un test suivant la procédure rasterscan décrite dans la référence [6].

\subsection{Amorçage sur des points chauds}

L'endommagement sur des défauts des composants peut être accru par la présence de surintensités locales dans les faisceaux. Celles-ci peuvent conduire, par autofocalisation à petite échelle (effet nonlinéaire Kerr), à un endommagement volumique sous forme de filaments ou à un endommagement systématique sur la face de sortie des composants. Cette occurrence est d'autant plus importante que l'épaisseur du composant illuminé est grande, c'est par exemple le cas des hublots des chambres d'expériences de type LIL et LMJ. Bercegol [9] a montré que l'endommagement par effet Kerr est limité lorsque le produit de l'intensité du faisceau incident par l'épaisseur du composant est inférieur à $20 \mathrm{GW} / \mathrm{cm}$ (à $351 \mathrm{~nm}$ ). Cette valeur permet de dimensionner les installations, soit en adaptant l'épaisseur des composants (lorsque cela est possible) connaissant les niveaux d'intensité, soit en limitant l'intensité en entrée du composant pour des épaisseurs données. 


\section{MESURE DE LA CROISSANCE DES DOMMAGES}

\subsection{Problématique}

Il est connu depuis le début des années 2000, qu'une fois amorcés, les dommages peuvent grossir lors d'illuminations successives, c'est-à-dire tir après tir. Cela est communément appelé la croissance des dommages et c'est le facteur qui limite la durée de vie des optiques ou qui modifie le profil d'emploi d'une installation laser de forte puissance. Au-delà d'un seuil, appelé seuil de croissance des dommages $\left(F_{\text {seuil }}\right)$ et qui est de l'ordre de $5 \mathrm{~J} / \mathrm{cm}^{2}$ pour la silice à $351 \mathrm{~nm}$, la croissance des dommages sur la face de sortie des optiques suit un comportement de type exponentiel : en seulement quelques tirs un dommage initialement micrométrique va atteindre une taille centimétrique. La surface utile sur l'optique est réduite, limitant l'énergie transmise et dégradant la qualité du faisceau. Cela se traduit par une qualité du faisceau focalisé sur la cible dégradée. De plus, en aval de la chaîne, ces dommages vont créer des surintensités dans le faisceau qui peuvent également dégrader la tenue au flux laser des composants en aval.

\subsection{Seuil de croissance et coefficients de croissance}

Sur la figure 2 sont reportés les coefficients de croissance mesurés lors d'une étude paramétrique de la croissance pour différentes durées d'impulsion et avec différentes modulations de phase. La croissance étant exponentielle, ces coefficients sont donnés par le rapport logarithmique de la surface du dommage $(S)$ entre deux tirs successifs : $k=\ln \left(S_{n} / S_{n-1}\right)$. De l'ensemble des données expérimentales, le seuil de croissance est situé autour de $5 \mathrm{~J} / \mathrm{cm}^{2}$, valeur couramment citée dans la littérature. Le coefficient de croissance prend ensuite des valeurs croissantes avec la fluence vue par les dommages. Les coefficients ont des valeurs dispersées autour d'une valeur moyenne traduisant un comportement erratique de la croissance tir à tir. En effet la phénoménologie de la croissance est très dépendante de la morphologie des dommages et plus précisément de la géométrie des fractures sous le dommage. Ce sont ces fractures qui absorbent le faisceau laser, l'absorption étant dépendante de la taille et de l'inclinaison des fractures [5].

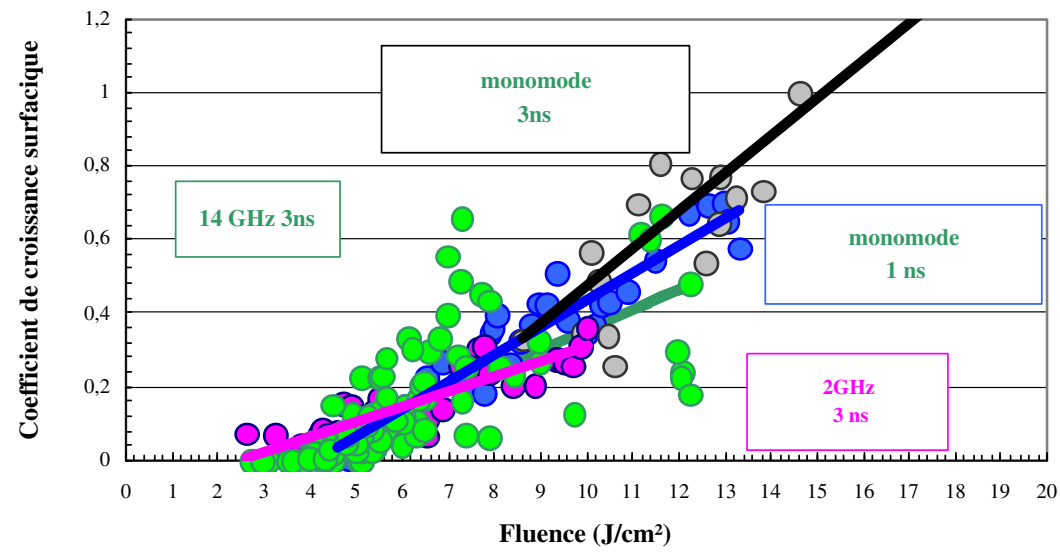

Figure 2. Coefficients de croissance des dommages sur la face de sortie de lames de silice. Les résultats sont légèrement dépendants de la durée d'impulsion et des modulations de phase. 


\section{EFFET DE LA COMBINAISON DES LONGUEURS D'ONDE}

\subsection{Problématique}

Certains composants d'une chaine laser sont illuminés par plusieurs faisceaux, de longueurs d'onde différentes, simultanément. Il s'agit par exemple des cristaux convertisseurs de fréquence. Le cas d'un cristal tripleur est un exemple concret : la troisième harmonique du laser est obtenue par combinaison de la longueur d'onde fondamentale et de sa seconde harmonique. Localement dans le cristal, le volume est alors éclairé simultanément par les trois longueurs d'onde. Puis en aval du cristal tripleur, les 3 faisceaux illuminent l'optique suivante, pour la LIL et le LMJ, le réseau focalisant.

\subsection{Cas des cristaux convertisseurs de fréquence}

Reyné a montré [10] l'effet coopératif du couplage des longueurs d'onde sur l'amorçage des dommages dans le volume d'un cristal tripleur. La combinaison d'impulsions à 1 et $3 \mathrm{w}$ induit un endommagement plus important que celui qui serait obtenu en considérant la résultante des deux endommagements pris séparément. Ces mesures sont dépendantes de la polarisation des faisceaux de tests [11], c'est-à-dire que les mesures doivent être réalisées suivant les configurations des cristaux telles que sur chaîne.

\subsection{Cas de la silice}

Le cas de l'amorçage et de la croissance des dommages pour des composants en silice est similaire. Des mesures en laboratoire pour ces deux étapes d'endommagement ont également montré un effet coopératif [12] : les densités de dommages mesurées en combinant plusieurs longueurs d'onde sont supérieures à la somme des densités prises séparément. Pour la croissance, la valeur du coefficient de croissance augmente également traduisant le fait que le faisceau $1 \mathrm{w}$ est également absorbé et participe à la croissance aussi efficacement que le $3 \mathrm{w}$.

\section{OBSERVATION SUR CHAINE ET PRÉVISION DE LA DURÉE DE VIE DES OPTIQUES}

\subsection{Problématique}

Il est nécessaire de suivre et de prédire l'endommagement sur une chaîne laser de puissance. Le suivi est nécessaire pour connaître le niveau de dégradation de l'optique, s'assurer que l'intégrité de la chaîne est préservée. A cette fin, des photographies des composants de la fin de chaîne sont effectuées. Le traitement des images permet de connaître le pourcentage de surface obturée et indirectement la transmission de l'optique. Cette dernière donnée déclenche alors la maintenance du composant lorsque le niveau de dégradation dépasse un critère d'endommagement dépendant de l'optique observée.

Au-delà de l'observation des composants, il est nécessaire de prédire l'endommagement des optiques sur la chaîne afin de programmer au mieux l'enchaînement des tirs et anticiper les maintenances.

\subsection{Modélisation de l'endommagement d'une chaîne de puissance}

La modélisation permet donc de prédire le comportement de l'optique sur la chaîne. Les données d'entrée pour correctement prédire cet endommagement sont :

- le champ proche du faisceau sur le composant analysé,

- la loi d'amorçage des dommages $D(F)$, traduisant la densité de dommages en fonction des niveaux de fluence dans le faisceau (Fig. 1). Couplé au champ proche, il peut être prédit le nombre et la position des dommages. 

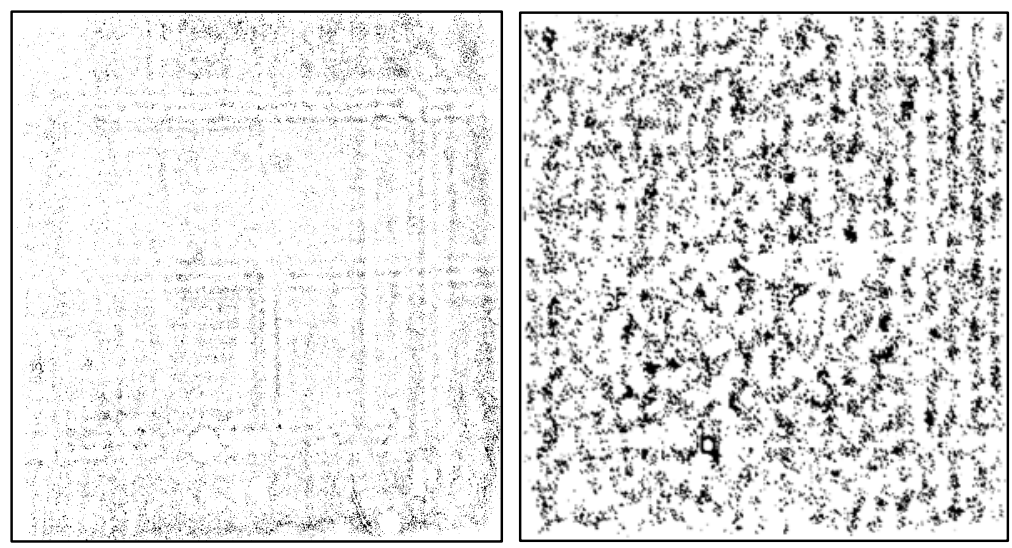

Figure 3. Comparaison entre une photographie d'un composant prise sur chaîne (à gauche) et le résultat de la modélisation avec les données d'entrée décrites au paragraphe 5.2. (à droite).

- les lois de croissance des dommages $k(F)$ (Fig. 2) qui tir après tir, une fois le dommage amorcé vont permettre de prédire la taille du dommage pour toute une séquence de tirs donnée.

- la séquence de tirs (l'énergie de chaque tir).

Sur la figure 3, une bonne correspondance est obtenue entre une photographie prise sur chaîne d'un composant à l'issue d'une série de tirs, et la prédiction de l'endommagement sur ce même composant avec la même série de tirs et les données d'entrée pour la modélisation. Notamment, la modélisation permet de connaître le pourcentage de surface obturée, donnée qui est en bonne adéquation avec les informations issues du traitement d'image de la photographie.

La durée de vie d'une optique sur chaîne peut être largement augmentée soit par une mise en forme spatiale spécifique à l'entrée de la chaîne laser, par l'application de pastille obturant le faisceau au niveau des dommages les plus gros soit en « réparant » les dommages par une procédure de refonte locale des dommages par laser $\mathrm{CO}_{2}$ [13]. Cette refonte permet de supprimer les fractures profondes à l'origine de la croissance et par conséquent d'augmenter considérablement la durée de vie de l'optique.

\section{CONCLUSION}

La connaissance des mécanismes d'endommagement permet de réduire les niveaux de densité de dommages sur les optiques, par amélioration continue de la qualité des optiques à travers l'optimisation des étapes de polissage ou par application de post-traitement de surface. Si malgré tout de rares défauts conduisent à la présence de quelques dommages sur l'optique, la phénoménologie de la croissance des dommages a conduit à bloquer la croissance par des procédures de stabilisation des dommages et par des mises en forme spatiale spécifiques des faisceaux. Dans le même temps, l'amélioration de la qualité des faisceaux conduit à réduire l'endommagement lié aux surintensités à petite échelle dans les faisceaux.

C'est principalement à partir de mesures en petits faisceaux et en laboratoire que les lois d'endommagement des optiques sont déterminées, il s'agit des lois d'amorçage des dommages $D(F)$ et des lois de croissance $k(F)$. Ce sont les données d'entrée qui permettent de modéliser et prévoir l'endommagement laser sur une chaîne de puissance, prévisions qui sont nécessaires pour l'évaluation du coût d'exploitation d'une chaîne laser et la gestion de son profil d'emploi. 


\section{Références}

[1] H. Bercegol, A. Boscheron, J.M. DiNicola, E. Journot, L. Lamaignère, J. Néauport and G. Razé, J. Phys. Conf. Ser. 112, (2008) 032013

[2] J. Néauport, L. Lamaignère, H. Bercegol, F. Pilon and J.C. Birolleau, Opt. Express 13, (2005) 10163

[3] F. Bonneau, P. Combis, J.L. Rullier, J. Vierne, M. Pelli, M. Savina, M. Broyer, E. Cottancin, J. Tuaillon, M. Pellarin, L. Gallais, J.Y. Natoli, M. Perra, H. Bercegol , L. Lamaignère, M. Loiseau and J. Donohue, Appl. Phys. B 75, (2002) 803

[4] J. Neauport, P. Cormont, L. Lamaignère, C. Ambard, F. Pilon and H. Bercegol, Optics Commun. 281, (2008) 3802

[5] H. Bercegol and P. Grua, Proc. SPIE 7132, (2008) p.71321B

[6] L. Lamaignère, S. Bouillet, R. Courchinoux, T. Donval, M. Josse, J.C. Poncetta and H. Bercegol, Rev. Sci. Instr. 78, (2007) 103105

[7] L. Lamaignère, M. Balas, R. Courchinoux, T. Donval, J. C. Poncetta, S. Reyné, B. Bertussi, and H. Bercegol, J. Appl. Phys. 107, (2010) 023105

[8] L. Lamaignère, T. Donval, M. Loiseau, J.C. Poncetta, G. Razé, C. Meslin, B. Bertussi and H Bercegol, Meas. Sci. Technol. 20, (2009) 095701

[9] H. Bercegol, L. Lamaignère, V. Cavaro, M. Loiseau, Proc. of SPIE 5991, (2006) p. $59911 \mathrm{Z}$

[10] S. Reyné, G. Duchateau, J.-Y. Natoli, and L. Lamaignère, Appl. Phys. Lett 96, (2010) 121102

[11] S. Reyné, G. Duchateau, J.Y. Natoli and L. Lamaignère, Opt. Express 17, (2009) 21652

[12] L. Lamaignère, S. Reyne, M. Loiseau, J.C. Poncetta, and H. Bercegol, Proc. of SPIE 6720, (2007) $67200 \mathrm{~F}$

[13] S. Palmier, L. Gallais, M. Commandré, P. Cormont, R. Courchinoux, L. Lamaignère, J.L. Rullier and P. Legros, Appl. Surf. Sci. 255, (2009) 5532 